\title{
Exotic and excited-state meson spectroscopy and radiative transitions from lattice QCD
}

\author{
Christopher E. Thomas ${ }^{1)}$ (Hadron Spectrum Collaboration) \\ Jefferson Laboratory, 12000 Jefferson Avenue, Suite \#1, Newport News, VA 23606, USA
}

\begin{abstract}
We discuss recent progress in extracting the excited meson spectrum and radiative transition form factors using lattice QCD. We mention results in the charmonium sector, including the first lattice QCD calculation of radiative transition rates involving excited charmonium states, highlighting results for high spin and exotic states. We present recent results on a highly excited isovector meson spectrum from dynamical anisotropic lattices. Using carefully constructed operators we show how the continuum spin of extracted states can be reliably identified and confidently extract excited states, states with exotic quantum numbers and states of high spin. This spectrum includes the first spin-four state extracted from lattice QCD. We conclude with some comments on future prospects.
\end{abstract}

Key words Lattice QCD, exotics, mesons, spectroscopy

PACS 12.38.Gc, 14.40.Be, 14.40.Rt

\section{Introduction}

A key ingredient in understanding the strong interaction is the calculation of the spectrum of QCD, along with the transition rates between different states, and testing these against experimental data. There has recently been a resurgence of interest in the charmonium system with the B-factories, CLEO-C, and BES finding missing states, making more accurate measurements of the properties of these states and discovering a number of new resonances that are not easily explained by the quark model. This has caused much theoretical speculations as to whether these states are hybrids or multiquark/molecular mesons. However, to date there has been no observation of a charmonium state having manifestly exotic $J^{P C}$, i.e. quantum numbers which can not result from a fermion-antifermion pair, such as $1^{-+}, 0^{+-}, 2^{+-}$. The upcoming GlueX, BES III and PANDA experiments will continue this work, probing the spectroscopy of both light and charm meson sectors.

Lattice QCD provides an $a b$ initio method for determining the hadron spectrum. An important aim of the Hadron Spectrum Collaboration is to use lattice QCD to extract the light meson spectrum and photocouplings. These calculations will be relevant for, among other things, the GlueX experiment at the JLab $12 \mathrm{GeV}$ upgrade where exotic mesons will be searched for in photoproduction. To this end, a program of work has been undertaken, first testing techniques for extracting excited spectra $[1,2]$ and photocouplings $[3,4]$ in the charmonium system which is computationally less demanding and where there is a profusion of experimental data. These methods are now starting to be applied to the spectrum of lighter mesons [5] and these proceedings give some highlights of recent progress, including a method for reliably identifying the spin of extracted states.

In Section 2 we highlight some results on excited radiative transitions in the charmonium sector and in Section 3 we present recent results on a highly excited and exotic isovector meson spectrum. We conclude in Section 4 with comments on future prospects.

\section{Charmonium Radiative Transitions}

Radiative transitions in charmonium can have significant branching ratios because there are no OZI allowed strong decays below open-charm threshold, and so there is a wealth of experimental data on these transitions. Recently, the first lattice QCD calculation of charmonium radiative transition rates involving states of high spin and exotics has been

Received XX December 2009

1) E-mail: thomasc@jlab.org

(C)2009 Chinese Physical Society and the Institute of High Energy Physics of the Chinese Academy of Sciences and the Institute of Modern Physics of the Chinese Academy of Sciences and IOP Publishing Ltd 
performed[4]. That reference used three-point correlation functions and a previous spectrum analysis $[1$, 2 ] to extract radiative transition rates and multipole amplitudes from quenched anisotropic lattices. Only connected diagrams were considered. The quenched approximation, where the light-quark degrees of freedom have been neglected, allows rather direct comparison to simple quark potential models. We refer to Ref. [4] for calculational details, results, systematic uncertainties, more detailed phenomenological discussion and comparison with models.

One highlight of this study is the calculation of the exotic $\eta_{c 1}\left(1^{-+}\right)$radiative decay. This decay, dominated by the magnetic dipole amplitude, was found to be significant: $\Gamma\left(\eta_{c 1} \rightarrow J / \psi \gamma\right) \sim 100 \mathrm{keV}$. This is of the same scale as many measured conventional charmonium transitions. However, it is significantly larger than conventional magnetic dipole transitions (c.f. $\Gamma\left(J / \psi \rightarrow \eta_{c} \gamma\right) \sim 2 \mathrm{keV}$ ), supporting the identification of this state as a spin-triplet hybrid[4]. We note that in a quenched lattice calculation, where there are no light-quark degrees of freedom, a state at this mass can not be a multiquark or molecular state.

The study of transitions involving excited vector charmonia found a significant signal for a nonexotic vector hybrid candidate in a magnetic dipole transition: $\Gamma\left(Y_{\text {hyb? }} \rightarrow \eta_{c} \gamma\right)=42(18) \mathrm{keV}$. This rate is again larger than conventional magnetic dipole transitions in charmonium, supporting the identification of this state as a spin-singlet hybrid; this assignment was suggested by the earlier spectrum analysis.

In addition, clear signals were found for electric dipole and magnetic quadrupole form factors in $\chi_{c 2} \rightarrow J / \psi \gamma$ and the hierarchy of multipole amplitudes allowed identification of the excited $\chi_{c 2}$ states as $2^{3} P_{2}$ and $1^{3} F_{2}$.

In summary, Ref. [4] has shown that these techniques can be used to successfully extract radiative transition rates from lattice QCD. The numerical results are subject to lattice systematic errors, such as those arising from a finite volume, a finite lattice spacing and the quenched approximation, but systematic improvements can be made as discussed in that reference. In the following section we discuss the first steps towards applying these technique to the light meson sector with unquenched lattices.

\section{Isovector Meson Spectrum}

In lattice QCD, the spectrum can be extracted from the two-point correlation functions of field operators which have the quantum numbers under in- vestigation. Access to states of spin-two and higher requires spatially separated quark fields, i.e. operators containing derivatives in the continuum. To enable efficient calculation of two-point functions containing such operators, a new quark-field construction algorithm, called "distillation", was recently developed[6].

Lattice QCD calculations consider the theory discretised on a four-dimensional Euclidean hypercubic grid. The three-dimensional rotational symmetry of the continuum is hence reduced to that of the $\mathrm{cu}-$ bic symmetry group. Spin no longer identifies irreducible representations of the cubic symmetry group, instead there are only five single-cover lattice irreps for each parity and charge conjugation $\left(A_{1}, T_{1}, T_{2}\right.$, $\left.E, A_{2}\right)[7]$. This introduces complications when studying particles of a particular spin. The distribution of the various components of a spin-J meson into the lattice irreps is known as subduction, the result of which is shown in Table 1. In the continuum limit, where full rotational symmetry is restored, the components subduced into different irreps will be degenerate, whereas at finite lattice spacing they will be split by an amount proportional to at least one power of the lattice spacing (e.g. the components of the spintwo state subduced in to the $T_{2}$ and $E$ irreps).

Table 1. Continuum spins subduced into lattice irreps $\Lambda(\operatorname{dim})[5]$

\begin{tabular}{ll}
$J$ & irreps \\
\hline 0 & $A_{1}(1)$ \\
1 & $T_{1}(3)$ \\
2 & $T_{2}(3) \oplus E(2)$ \\
3 & $T_{1}(3) \oplus T_{2}(3) \oplus A_{2}(1)$ \\
4 & $A_{1}(1) \oplus T_{1}(3) \oplus T_{2}(3) \oplus E(2)$
\end{tabular}

The above discussion suggests that a simple method of assigning continuum spins would be to identify degeneracies across lattice irreps according to the subduction patterns shown in Table 1. However, there are many approximate degeneracies in the spectrum and so it is easy to confuse these with those arising from discretisation. For example, the $\chi_{c 0,1,2}$ triplet of states in charmonium is split only by the small spin-orbit interaction. These states would appear as a state in each of the irreps $A_{1}^{++}, T_{1}^{++}, T_{2}^{++}$ and $E^{++}$, which is the same pattern as a spin-four state split by discretisation effects.

Ref. [5] describes a method for carefully constructing operators out of a gamma matrix and spatial covariant derivatives which allows the continuum spin of extracted states to be reliably identified. We refer to that reference for details of the method and results; 
here we briefly review the salient points and highlight some results.

For a first investigation, Ref. [5] used the threeflavour degenerate-quark-mass dataset with unphysically heavy light quarks on unquenched anisotropic lattices $[8,9]\left(m_{\pi}=m_{K}=m_{\eta} \approx 700 \mathrm{MeV}\right)$, with lattice spacings $a_{s} \sim 0.12 \mathrm{fm}, a_{t}^{-1} \sim 5.6 \mathrm{GeV}$ and a spatial lattice extent of $\sim 2 \mathrm{fm}$. Only connected Wick contractions were computed, giving access to isovector quantum numbers.

The spectrum was extracted using a large basis of operators and a variational method[10, 11]. The extracted spectrum across lattice irreps, using all operators with up to three derivatives, is shown in Figs. 1 and 2. States with exotic quantum numbers are highlighted. In these figures, the number of operators in each irrep is given below the irrep label and all masses are scaled by the $\Omega$ baryon mass as extracted on this lattice [9]. Boxes represent the extracted mass and one sigma statistical uncertainties. Color coding indicates continuum spin identification described below; orange boxes have well determined masses but undetermined spin whereas grey boxes have masses that are not well determined by the variational fitting method.

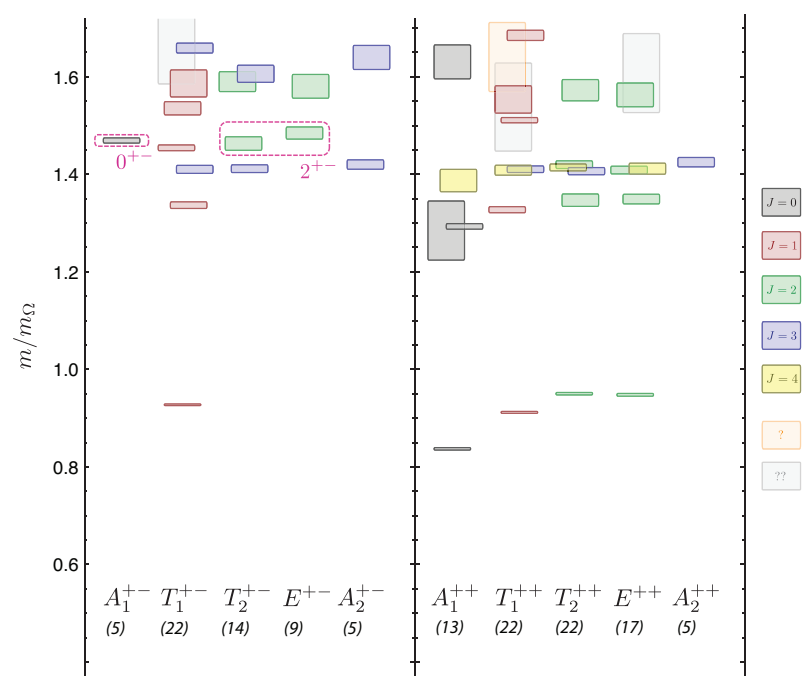

Fig. 1. From Ref. [5]. Extracted spectrum of states in the $P C=+-,++$ channels displayed by lattice irrep. States with exotic quantum numbers $0^{+-}$and $2^{+-}$are highlighted. More details are given in the text.

In the continuum limit, the operators, $\mathcal{O}^{J, M}$, of Ref. [5] are of definite spin such that $\left\langle 0\left|\mathcal{O}^{J, M}\right| J^{\prime}, M^{\prime}\right\rangle=Z^{[J]} \delta_{J, J^{\prime}} \delta_{M, M^{\prime}}$ and so $\left\langle 0\left|\mathcal{O}_{\Lambda, \lambda}^{[J]}\right| J^{\prime}, M\right\rangle=\mathcal{S}_{\Lambda, \lambda}^{J, M} Z^{[J]} \delta_{J, J^{\prime}} . \quad \mathcal{S}_{\Lambda, \lambda}^{J, M}$ describes the subduction of an operator with continuum spin $J$ (component $M$ ) on to lattice irrep $\Lambda$ (row $\lambda$ ). $Z^{[J]}$ is a single number of dynamical origin describing the overlap of the state of spin $J$ onto the operator used. As described in Ref. [5], these overlaps can be used to identify the spin of the extracted states. Firstly, the relative magnitudes of the extracted $Z$ values can be considered and it is found that each state has large overlap only onto operators of a single spin; Figure 3 shows an example of this for the $J^{--}$mesons. Secondly, states are matched across difference irreps and the $Z$ values of common operators subduced across irreps are compared. At finite lattice spacings small deviations from equality are expected. Ref. [5] has shown that these $Z$ values agree well across irreps.

Figs. 1 and 2 show that this method has enabled the extraction of many excited states across all $P C$ combinations and the confident assignment of continuum spin. Spin-four states have been identified in lattice QCD for the first time: $4^{++}, 4^{-+}$and $4^{--}$. States with exotic quantum numbers $\left(0^{+-}, 1^{-+}, 2^{+-}\right)$ have been extracted, hinting at the influence of gluonic degrees of freedom. In addition, Fig. 3 shows that the third excited vector state (marked with $\dagger$ ) has a qualitatively different pattern of $Z$ values compared to the lower lying vector states. Distinctively, there is a large overlap on to an operator containing $D_{J=1}^{[2]}$, proportional to the commutator of two covariant derivatives. This commutator vanishes in the absence of a gluonic field and so hints at a gluonic component, suggesting the identification of this state as a crypto-exotic vector hybrid. The overlaps on to other operators suggest some mixing with a conventional vector state.

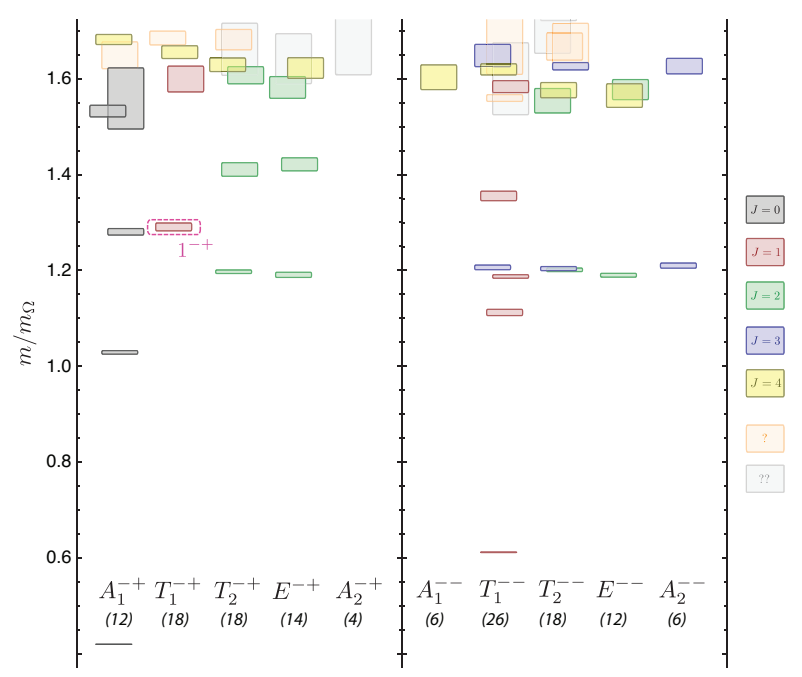

Fig. 2. From Ref. [5]. As previous figure but for $P C=-+,--$. The lowest lying exotic $1^{-+}$is highlighted. 


$$
\begin{aligned}
& J=0 \quad \square\left(a_{1} \times D_{J=1}^{[1]}\right)^{J=0} \square\left(a_{1} \times D_{J_{13}=2, J=1}^{[3]}\right)^{J=0} \\
& J=1 \square(\rho)^{J=1} \square\left(a_{1} \times D_{J=1}^{[1]}\right)^{J=1} \square\left(\rho \times D_{J=2}^{[2]}\right)^{J=1} \square\left(\pi \times D_{J=1}^{[2]}\right)^{J=1} \\
& J=2 \square\left(a_{1} \times D_{J=1}^{[1]}\right)^{J=2} \square\left(\rho \times D_{J=2}^{[2]}\right)^{J=2} \square\left(a_{1} \times D_{J_{13}=2, J=3}^{[3]}\right)^{J=2} \\
& J=3 \quad\left(\rho \times D_{J=2}^{[2]}\right)^{J=3} \square\left(a_{0} \times D_{J_{13}=2, J=3}^{[3]}\right)^{J=3} \square\left(a_{1} \times D_{J_{13}=2, J=3}^{[3]}\right)^{J=3} \\
& J=4 \quad\left(a_{1} \times D_{J_{13}=2, J=3}^{[3]}\right)^{J=4}
\end{aligned}
$$
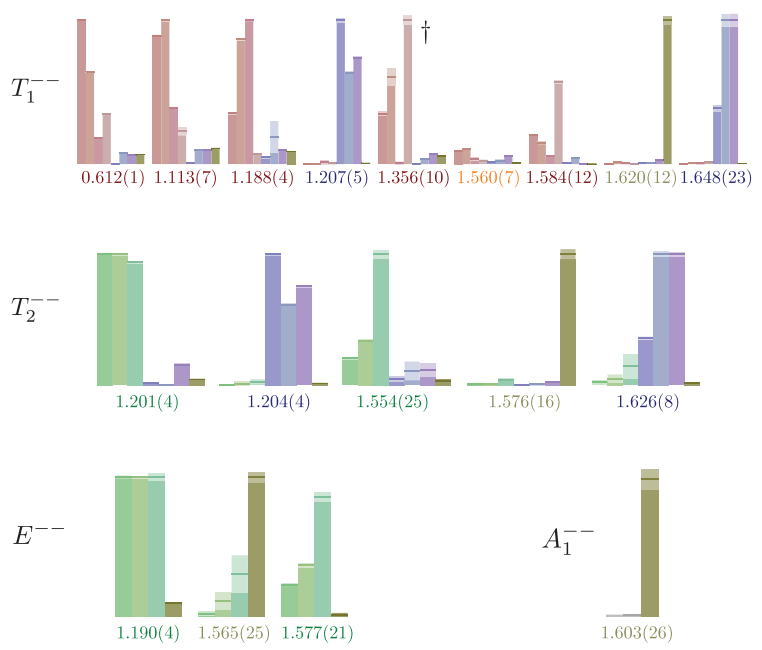

Fig. 3. From Ref. [5]. Overlaps, $Z$, of a selection of operators onto states labelled by $m / m_{\Omega}$ in each lattice irrep, $\Lambda^{--}$. $Z$ 's are normalised so that the largest value across all states is equal to 1 . Lighter area at the head of each bar represents the one sigma statistical uncertainly.

The extracted spectrum has features of the $n^{2 S+1} L_{J}$ classification from quark models, along with states that do not fit in to that pattern. However, there is no sign of an abundance of two-meson states which might be expected above $2 m_{\pi} \sim 0.85 m_{\Omega}$. For example, a pseudoscalar-vector state with minimal allowed relative momentum in the finite box would be expected in the $A_{1}^{--}$channel close to $1.2 m_{\Omega}$. However, the lightest state extracted in that channel is a spin-four state above $1.5 m_{\Omega}$. A similar calculation on a lattice with a larger spatial extent $(\sim 2.4 \mathrm{fm})$ results in an identical spectrum, within statistical fluctuations, to that shown above. This gives further evidence that there are no two-meson states in the spectrum: a change in volume would cause the allowed relative momentum, and hence energy levels, to change considerable. An argument as to why no two-meson states are extracted at this unphysically large pion mass is given in Ref. [5].

In summary, the method of Ref. [5] has been successful in extracting a full spectrum of isovector mesons in QCD including highly excited mesons and exotic and non-exotic hybrid mesons.

\section{Conclusions}

We have discussed recent progress in extracting highly excited spectra and calculating excited radiative transitions using lattice QCD. The results described here are just the start of ongoing work; this is now progressing to study the spectrum of light mesons on different volumes and at various lower pion masses (where the strange quark is no longer degenerate with the up and down quarks). Another future direction is the study of multi-meson states by constructing multi-meson operators and a long term aim is the extraction light meson photocouplings, with particular interest in exotic states.

I thank Jo Dudek, Robert Edwards, Michael Peardon, David Richards and other colleagues in the Hadron Spectrum Collaboration. The Chroma software suite [12] was used to perform this work on clusters at Jefferson Laboratory using time awarded under the USQCD Initiative. Authored by Jefferson Science Associates, LLC under U.S. DOE Contract No. DE-AC05-060R2317\%. The U.S. Government retains a non-exclusive, paid-up, irrevocable, worldwide license to publish or reproduce this manuscript for U.S. Government purposes.

\section{References}

1 J. J. Dudek, R. G. Edwards, N. Mathur, and D. G. Richards, Phys. Rev. D77, 034501 (2008), arXiv:0707.4162 [hep-lat].

2 J. J. Dudek and E. Rrapaj, Phys. Rev. D78, 094504 (2008), arXiv:0809.2582 [hep-ph].

3 J. J. Dudek, R. G. Edwards, and D. G. Richards, Phys. Rev. D73, 074507 (2006), arXiv:hep-ph/0601137.

4 J. J. Dudek, R. Edwards, and C. E. Thomas, Phys. Rev. D79, 094504 (2009), arXiv:0902.2241 [hep-ph].

5 J. J. Dudek, R. G. Edwards, M. J. Peardon, D. G. Richards, and C. E. Thomas, arXiv:0909.0200 [hep-ph], accepted for publication in Phys. Rev. Lett.
6 Hadron Spectrum, M. Peardon et al., Phys. Rev. D80, 054506 (2009), arXiv:0905.2160 [hep-lat].

7 R. C. Johnson, Phys. Lett. B114, 147 (1982).

8 R. G. Edwards, B. Joo, and H.-W. Lin, Phys. Rev. D78, 054501 (2008), arXiv:0803.3960 [hep-lat].

9 Hadron Spectrum, H.-W. Lin et al., Phys. Rev. D79, 034502 (2009), arXiv:0810.3588 [hep-lat].

10 M. Luscher and U. Wolff, Nucl. Phys. B339, 222 (1990).

11 B. Blossier, M. Della Morte, G. von Hippel, T. Mendes, and R. Sommer, JHEP 04, 094 (2009), arXiv:0902.1265 [hep-lat].

12 SciDAC Collaboration, R. G. Edwards and B. Joo, Nucl. Phys. B. Proc. Suppl. 140, 832 (2005), arXiv:heplat/0409003. 